\title{
EXPERIMENTO PARA LA ENSEÑANZA INTRODUCTORIA DE ECONOMÍA: EL EFECTO DECOY
}

\section{EXPERIMENT FOR ECONOMY INTRODUCTORY TEACHING: THE DECOY EFFECT}

\section{Víctor Eduardo Romero Cueva, Lic.}

Economista con mención en Gestión Empresarial (Ecuador). Docente del Curso de Nivelación de Carrera en la Universidad Técnica de Machala,

Ecuador. viromero@mgs.ecotec.edu.ec

Laura Amarilis Borja Herrera, Ph.D.

Doctora en Ciencias Contables y Empresariales (Perú). Vicerrectora Académica en la Universidad Técnica de Machala, Ecuador. Iborja@utmachala.edu.ec

Marlyn Elizabeth Luna Romero, Lic.

Licenciada en Bellas Artes Especialización en Artes Plásticas y Visuales (Ecuador). Docente del Curso de Nivelación en la Universidad Técnica de Machala, Ecuador. meluna_est@utmachala.edu.ec

\section{ARTÍCULO DE INVESTIGACIÓN}

Recibido: 17 de septiembre de 2019.

Aceptado: 25 de noviembre de 2019.

\section{RESUMEN}

En un mundo tan cambiante, hay paradigmas que se vuelven monótonos y que pasan a ser obsoletos. El artículo busca contribuir en la enseñanza introductoria de economía a través de un experimento social que analiza el comportamiento humano, el cual aplica el efecto Decoy para explicar cómo toman decisiones los individuos. El experimento fue aplicado a una muestra de 100 estudiantes del Curso de Nivelación de Carrera de la Universidad Técnica de Machala, a quienes se presentó diferentes escenarios de inversión donde se combinaba riesgo y rentabilidad. La presencia de sesgos cognitivos permitió desentrañar una propiedad 
básica de los seres humanos al momento de efectuar elecciones: la irracionalidad. Se concluyó que para un mejor entendimiento de la economía se debe tomar a los experimentos sociales como la base de la enseñanza, que, además, debe estar reforzada por los diversos postulados de pensamiento económico.

Palabras clave: economía, DECOY, experimento, comportamiento, decisiones.

\section{ABSTRACT}

In such a changing world, there are paradigms that become monotonous and become obsolete. The article seeks to contribute to the introductory teaching of economics through a social experiment that analyzes human behavior, which applies the Decoy effect to explain how individuals make decisions. The experiment was applied to a sample of 100 students of the Career Leveling Course of the Universidad Técnica de Machala, to whom different investment scenarios were presented where risk and profitability were combined. The presence of cognitive biases allowed to unravel a basic property of human beings at the time of making choices: irrationality. It was concluded that for a better understanding of the economy, social experiments should be taken as the basis of education, which, in addition, should be reinforced by the various postulates of economic thought.

Keywords: economy, DECOY, experiment, behavior, decisions.

\section{INTRODUCCIÓN}

Desde los albores del tiempo, hay un fenómeno que se repite en este planeta, hasta la época actual: la perpetuación de la especie. Una de ellas, ha tenido un comportamiento bastante singular, puesto que en la búsqueda por satisfacer sus necesidades y garantizar la supervivencia, descubrió el excedente: una alternativa para administrar los recursos escasos, incrementar la productividad y garantizar la satisfacción de demandas futuras. Es el momento clave de la Economía que derivó en el origen del mercado.

Stiglitz (1993) lo define:

La economía estudia el modo en que eligen los individuos, las empresas, el Estado y otras entidades de nuestra sociedad y en que esas elecciones determinan la manera en que se utilizan los recursos que tiene. El término escasez ocupa un lugar destacado en economía: las elecciones son importantes porque los recursos son escasos.

ISSN: 1390-9320, Edición Especial, diciembre 2019 
Comencemos con una operación elemental que reza:

$$
100 \%-80 \%=20 \%
$$

¿Es irrefutable? Sí, por supuesto. En España, Australia, Papúa Nueva Guinea, en cualquier rincón del orbe, recitarán al unísono el mismo resultado. A esto, mis queridos cursantes, se le llama ciencias exactas.

Pero, hay algo que fluctúa a diario en los mercados internacionales: el precio del petróleo.

Supongamos, por ejemplo, Nepal y Ecuador. ¿Cómo afectará a estas dos naciones una subida del precio internacional del crudo?

Para explicarlo, hay que mencionar la importancia vital del petróleo en la vida de un país situado entre China y la India, y que no tiene salida al mar. Ah sí, tampoco tiene producción petrolera. Muy probablemente, los nepalíes tengan que adquirir el energético por una cifra nada módica. Así pues, es evidente la relación directa entre el precio del crudo y su costo de vida.

En el caso de Ecuador (petrolero por excelencia), si el precio del crudo se dispara, muy probablemente aumenten las ofertas laborales, así como los beneficios e incentivos para ese sector. Y no es para menos, durante más de siglo y medio, el petróleo ha sido determinante para el desarrollo de los pueblos. Ahora, imaginen el siguiente escenario: Ecuador produciendo más de 500.000 barriles diarios y exportándolos a un precio atractivo. Muy probablemente, ocurra un incremento en la renta de los ecuatorianos. Así pues, es evidente la relación inversa entre el precio del crudo y su costo de vida.

\section{REVISIÓN TEÓRICA}

Hay una temática que se aborda hasta el hartazgo: enseñanza tradicional vs enseñanza moderna. Referido al segundo modelo, en la mayoría de los casos se habla de la importancia de incluir las TIC en el proceso educacional, así como también, involucrar con mayor frecuencia la praxis. Es decir, se sabe qué hacer, pero no precisamente cómo hacerlo.

Más específicamente, cuando se realiza la introducción a economía, dirigida a estudiantes, emprendedores o cualquier tipo de persona interesada en el campo; se encuentra que la palabra "dinero" es habitualmente mencionada. De hecho, muchos se inician creyendo que terminarán convirtiéndose en los próximos Robert Kiyosaki, Jordan Belfort o Chris Gardner, por citar algunos nombres de millonarios que el cine o los best-sellers se han encargado de popularizar. 
Esto deja entrever desde el principio la relación personal que tienen los sujetos con las ganancias o beneficios monetarios que, por cierto, no es una postura equivocada, pero sí algo imprecisa, puesto que el último fin de la economía es el bienestar humano.

Pero... ¿cómo se alcanza el bienestar humano? Haciendo elecciones racionales. El problema radica en que, generalmente, se hace lo contrario.

Así pues, se han desarrollado muchas corrientes de pensamiento que explican su accionar en un lugar llamado mercado.

Por ejemplo, en el siglo XIX, la Escuela Neoclásica de pensamiento económico presentaba una aproximación del Homo Sapiens conocida como Homo Economicus, la cual explicaba que los seres humanos buscan el máximo beneficio al menor costo.

Como experto en el área de Economía del Comportamiento, (Sugden, 2009) resume: "Traditionally, economic policy advice has been based on the assumption that individuals act rationally within whatever constraints they face."

En otras palabras, su racionalidad era guiada únicamente por incentivos económicos. Pero, hoy en día, se sabe que existen otros tipos de incentivos: sociales, morales e incluso subliminales.

Adam Smith, considerado el padre de la Economía Moderna y célebre por su obra "La Riqueza de las Naciones" (1776), había escrito años atrás, "La Teoría de los Sentimientos Morales" (1759), un libro que desentrañaba gran parte de la naturaleza de los seres humanos guiada también por los sentimientos. Así pues, durante mucho tiempo se ha modelizado erróneamente el comportamiento humano, el cual es individualista, como señala el economista escocés, pero no siempre está influenciado por estímulos monetarios.

Ciertamente hay capítulos de la obra de Smith que para la Escuela Neoclásica no fueron relevantes, pero que en pleno siglo XXI están cobrando interés debido a la forma en que se presenta la información.

Asimismo, lo asienten Pascale \& Pascale, 2007, pp.166:

En la economía neoclásica, los detalles de cómo funciona el cerebro humano en la toma de decisiones no fueron tomados en consideración. Los aportes cognitivos son sustanciales en el esclarecimiento de la toma de decisiones y así se expuso el pensamiento de Simon, Allais, Kahenman y Tversky. 


\section{Generalidades de la Economía del Comportamiento:}

Explicar la conducta de las personas o conocer los motivos que les llevan a tomar una decisión nunca fue tarea fácil. Al repasar las distintas teorías que subyacen en torno a esta temática, se encuentra una que se aleja de la racionalidad clásica.

Esto es, la "Prospect Theory" o Teoría Prospectiva, desarrollada por Daniel Kahneman y Amos Tversky que se expone a continuación en palabras de (Pascale y Pascale, 2007):

Dos fenómenos de este efecto se destacan en la citada teoría de los mencionados autores. Ellos son: aversión al riesgo y aversión a una pérdida segura.

La aversión al riesgo dice relación con el hecho que, psicológicamente los agentes económicos le otorgan más importancia a una pérdida que a una ganancia de la misma magnitud. Supónganse que una persona tiene iguales probabilidades, esto es $50 \%$ en cada caso de perder $\$ 60$ o ganar una determinada cifra. La pregunta es ¿cuánto tiene que ser el monto de la probable ganancia? \$150, \$250, \$500 o qué cifra? Numerosos estudios experimentales ubican a la ganancia que obtuvo más respuesta en torno a $\$ 150$. Ello significa que las personas valoran la pérdida de $\$ 60$ dos veces y media más que una ganancia probable $(\$ 150 / 60=2.5)$.

El segundo fenómeno de efecto enmarcamiento por Tversky ${ }^{1}$ y Kahneman es el de aversión a una pérdida segura. El mismo en este caso dice relación que los sujetos económicos aceptarán un curso de acción riesgoso en un intento por evitar una pérdida segura. Supongamos que un sujeto se ve enfrentado a optar por una pérdida segura de $\$ 70$ y una opción riesgosa. La alternativa riesgosa es una situación con el $70 \%$ de probabilidad de ganar $\$ 100$ y el $30 \%$ de ganar 0 . ¿Cuál será la opción que tomará? Esto es, ¿la pérdida segura o la opción riesgosa? La mayor parte los experimentos muestran a los sujetos inclinándose por la opción riesgosa.

Es así como se puso entredicho la idea de que el ser humano es fundamentalmente racional puesto que, como se indica en las pruebas, las elecciones realizadas por los sujetos

\footnotetext{
${ }^{1}$ Amos Tversky, había fallecido antes, sino indudablemente, como lo dejó entrever la Real Academia Sueca de Ciencias también habría recibido el Nobel de Economía que recibió Kahneman, por sus contribuciones sobre la investigación psicológica en la ciencia económica, en particular en lo que respecta al juicio humano y la toma de decisiones en condiciones de incertidumbre.
}

ISSN: 1390-9320, Edición Especial, diciembre 2019 
presentaron lo que también se conoce como racionalidad limitada o "bounded rationality"; un concepto propuesto por Herbert Simon².

Consecuentemente, Daniel Kahneman y Amos Tversky desarrollaron una teoría descriptiva de la toma de decisiones de los seres humanos. En su teoría resaltan expresamente que el hombre "común", tiene un modelo perceptivo estructurado de forma de conocer los cambios y las diferencias, más que valorar las dimensiones absolutas.

En una obra de arte, un valor puede parecer más luminoso según se coloque al lado de uno u otro color diferente.

La figura que sigue lo ilustra mejor:

Figura 1. Sesgos cognitivos en la percepción visual
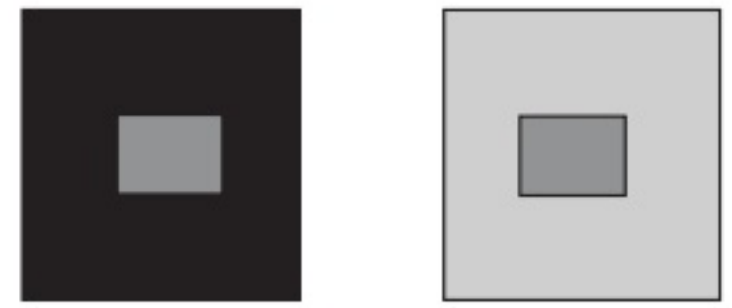

Fuente: (Pascale \& Pascale, 2007) en base a Kahneman (2002)

Como se puede apreciar, aunque el cuadrado pequeño tiene el mismo color, aparece con luminosidad distinta según en qué cuadrado mayor esté insertado. La búsqueda de una dependencia en una referencia en la toma de decisiones es lo que lleva a ambos autores a realizar esta analogía con los procesos perceptivos visuales.

Las diferencias de luminosidad son cuestiones relativas y no absolutas.

Sobre este apartado Kahneman (2002) señala:

"El valor está dado por la diferencia entre los estados económicos y no están dados por los datos presentado en sí mismos".

\footnotetext{
${ }^{2}$ Herbert Simon fue galardonado con el Nobel de Economía en 1978 por sus contribuciones sobre el proceso de toma de decisiones, especialmente en el campo administrativo.
} 
En la actualidad, la Economía conductual tiene grandes referentes, uno de ellos, Alain Samson" ${ }^{3}$, sostiene: "La economía conductual emplea experimentación psicológica para desarrollar teorías sobre toma de decisiones humanas y ha identificado un rango de sesgos como resultado de cómo piensan y sienten las personas" (Samson, 2014).

Como bien advierte (Tlapanco, 2016) en base a (Plott \& Smith, 2008):

Muchas preguntas de la teoría económica no pueden ser respondidas a través de experimentos. Las instituciones, entendidas como el conjunto de normas de acción, delimitan los resultados de las acciones de los individuos y estas pueden ser caracterizadas a través de los supuestos que se utilizan en la teoría económica. Los modelos económicos bien entendidos tendrán la respuesta para conocer el alcance de su capacidad explicativa y de aplicación.

Por esta razón se presenta el siguiente problema de investigación: ¿Cómo contribuir en la enseñanza introductoria de economía a través del comportamiento humano? En este sentido se presenta el objetivo general: explicar la toma de decisiones de los individuos mediante un experimento social para la enseñanza introductoria de economía

Ciertamente, no existe una fórmula mágica para educar en economía, pero a mediados de los años 40 en Estados Unidos, un profesor de Harvard, Edward H. Chamberlin utilizó un recurso desaprovechado en este campo: los experimentos. Los mismos que, bien diseñados, pueden servir para comprender el funcionamiento del mercado y, sobre todo, cómo toman decisiones los individuos. Utilizando como demandantes y oferentes a estudiantes que podían vender y comprar unos productos ficticios en el mercado, contrastó si se cumplía la predicción de que los mercados se equilibran al precio de mercado resultante. Su mercado, donde los estudiantes iban y venían negociando entre los pupitres, arrojó un resultado bastante sorprendente: se vendía una cantidad notablemente mayor de la predicha por los modelos teóricos (Chamberlin, 1948).

Tradicionalmente, la Economía se ha considerado una ciencia no experimental, pero para Vernon Smith, ganador del premio Nobel de Economía en 2002 y uno de los predecesores de la Economía Experimental, esta apreciación ha cambiado. Él fue uno de los estudiantes que participaron en los experimentos de Chamberlin y no quedó muy convencido de la interpretación que su profesor había hecho. Por lo cual, quince años más tarde publicó dos

\footnotetext{
${ }^{3}$ Alain Samson es fundador de BehavioralEconomics.com y Doctor en Psicología Social por la London School of Economics and Political Science.
} 
trabajos en los que se mostraba que con información pública los precios sí convergían al equilibrio (Smith, 1962).

Según Smith (2005) "La economía experimental aplica métodos de laboratorio para estudiar las interacciones de los seres humanos en los contextos sociales gobernados por reglas explícitas o implícitas"

García y Carreño (2012) en base a Vernon Smith, asienten:

Los individuos no son exactamente como el homo economicus que defendía la economía neoclásica. En ocasiones los individuos son mejores de lo que se esperaría en una competencia perfecta, y a veces, peores de lo que se esperaría en un ambiente de cooperación.

En Samuelson y Nordhaus (1985) se declara:

Una forma posible de descubrir leyes económicas... Es por medio de experimentos controlados... Los economistas [desafortunadamente]... no pueden realizar experimentos controlados como los químicos o los biólogos, porque no pueden controlar fácilmente los factores importantes. Como los astrónomos o los meteorólogos se deben contentar generalmente con observar.

"La economía experimental detecta los puntos débiles de los mercados y de las políticas antes de que las teorías sean grandes iniciativas públicas". Así pues, cabe destacar que la economía experimental está vinculada directamente con la microeconomía puesto que la mayoría de las decisiones estudiadas son individuales. Tal como lo menciona (Sugden, 2009): "It is only relatively recently that behavioural economists have started to think seriously about the implications of their findings for public policy".

"Los economistas son criticados a veces por el hecho de que la economía no es una ciencia. El comportamiento humano, dicen, no puede ser analizado con la misma objetividad que el de los átomos y las partículas... Más aún, no hay laboratorio en el cual los economistas puedan poner a prueba sus hipótesis." (Encyclopedia Britannica, 1991)

Como señala (Fatás \& Roig, 2004), los cuatro componentes básicos de todo experimento económico son: entorno, institución, comportamiento y resultados. 
Figura 2. Un experimento económico

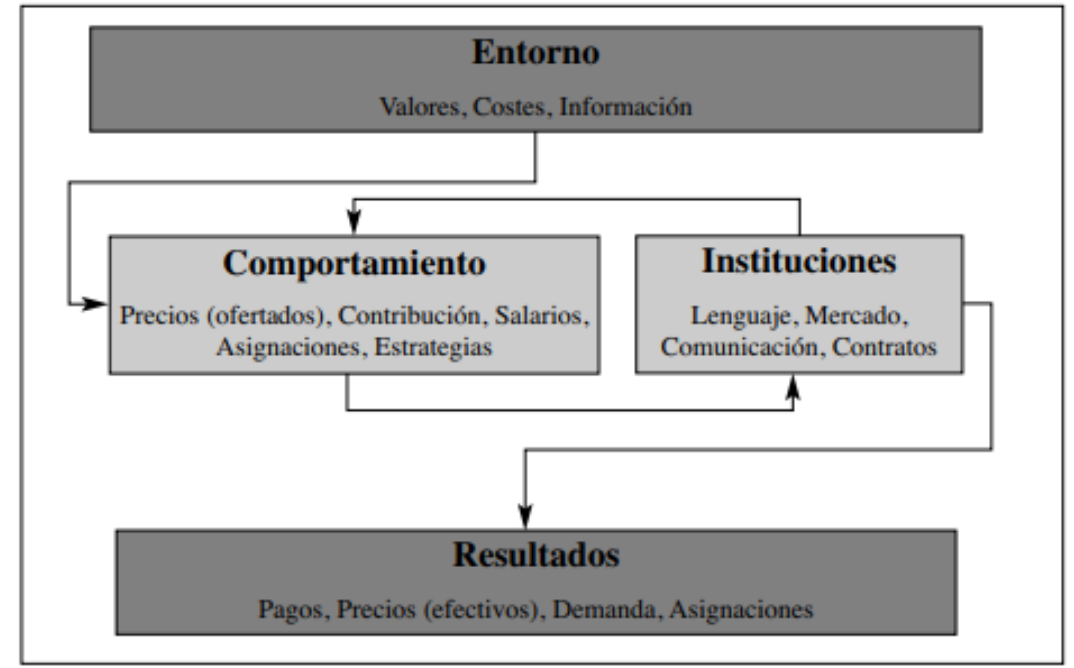

Fuente: (Fatás \& Roig, 2004)

El primero de ellos, entorno, se refiere a las características relevantes de los sujetos como dotación de recursos, preferencias, información. Es decir, una persona al ingresar como participante a un experimento posee características (edad, preferencias o gustos, conocimientos previos, etc.). Por otro lado, las instituciones se refieren a la forma como interactúan los agentes y definen los resultados derivados de estas interacciones (cuánto gana o pierde si toma una u otra alternativa, etc.). Las instrucciones, que básicamente le indican al sujeto participante en un experimento que puede hacer, son un reflejo de la institución que gobierna el experimento. El comportamiento se refiere a las decisiones o acciones observables de los sujetos y que se presentan como reacción al entorno y las instituciones. Por supuesto, todos los ejercicios experimentales generan resultados. En el caso de los experimentos se refieren a precios, cantidades demandadas $u$ ofrecidas, ganancias de vendedores o compradores, etc.

Por consiguiente, la economía experimental juega un rol determinante en la presente investigación, no se deduce otra cosa, sino que en un futuro no muy lejano el cambio de paradigma educativo será toda una realidad.

En virtud de ello, ciertas teorías económicas pueden ser estudiadas y probadas para una mejor aplicación en el mundo real. Al día de hoy, hay una gama amplia de recursos para fomentar la experimentación. Uno de ellos, muy presente en el diario vivir, pero que al mismo tiempo pasa desapercibido es el efecto Decoy. 


\section{Efecto Decoy}

También conocido como "efecto de dominio asimétrico", es un fenómeno que modifica la conducta de los consumidores, quienes tienden a cambiar su preferencia entre dos alternativas cuando también se incluye una tercera.

Para clarificarlo, (Huber, Payne, \& Puto, 1982) afirman: "An asymmetrically dominated alternative is dominated by one item in the set but not by another. Adding such an alternative to a choice set can increase the probability of choosing the item that dominates it."

Figura 3. Posición de la opción asimétricamente dominada decoy

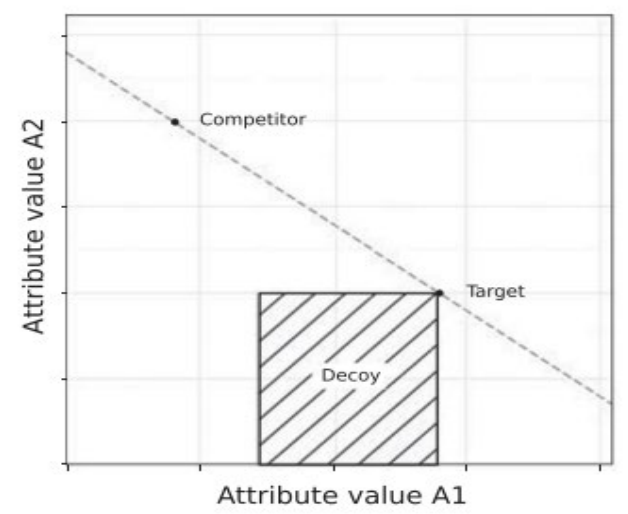

Fuente: (Kaptein, Emden, \& Lanuzzi, 2016) en base a (Huber, Payne, \& Puto, 1982)

Dicho, en otros términos, una alternativa dominada asimétricamente está dominada por un elemento del conjunto; pero no por otro. Agregar tal alternativa (es decir, el Decoy) a un conjunto de opciones permite incrementar la probabilidad de elegir el elemento que le domina (es decir, el target)

En palabras de (González \& Lordan, 2013):

La opción Decoy no se constituye como una alternativa válida dentro del conjunto de opciones, sino que su utilidad radica en modificar las preferencias y el atractivo percibido por el usuario de, al menos, una de las opciones originales presentes en el conjunto.

Adicionalmente, resulta sustancial conocer las distintas estrategias o posiciones que puede adoptar una opción Decoy que, de hecho, son factores que pueden incrementar la posibilidad de elegir el target. 
En esta literatura, (Kaptein, Emden, \& Lanuzzi, 2016) lo indican:

The range decoy $(\mathbf{R})$ is defined as an option, that is slightly weaker than the target on the target's weakest attribute: a range decoy increases the range of an attribute dimension on which the competitor is weakest. The frequency decoy (F) refers to an option that increases the frequency of the attribute dimension on which the target is superior. The range-frequency decoy (denoted RF) is a combination of range and frequency manipulations, and the extreme range $\left(\mathbf{R}^{\star}\right)$ decoy represents a more extreme version of the range decoy.

Figura 4. Estrategias de posición - opción decoy

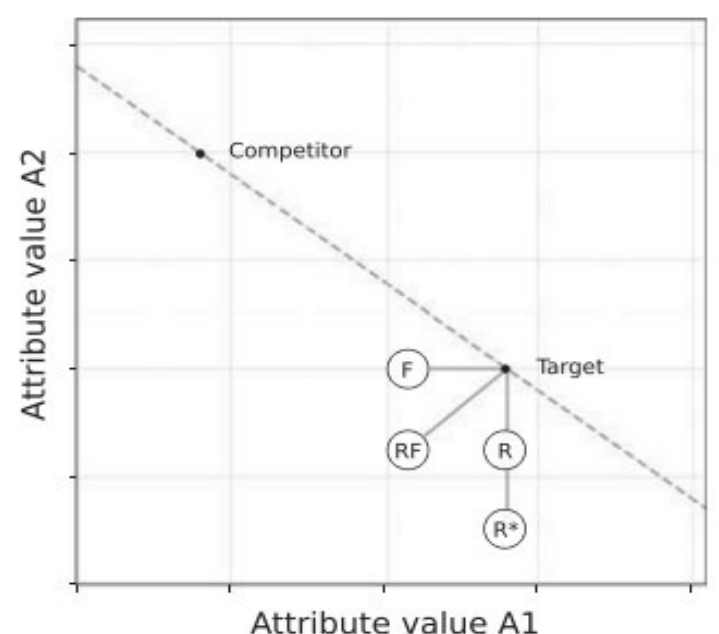

Fuente: (Kaptein, Emden, \& Lanuzzi, 2016) en base a (Huber, Payne, \& Puto, 1982)

A simple vista, pareciera ser que para incrementar el atractivo de cierta alternativa (target) cuando rivaliza con otra (competitor), bastaría con agregar una tercera (decoy). Aunque este procedimiento sea teóricamente correcto, podría no tener un tamaño de efecto esperado, ya que la probabilidad de elegir el target está influenciada también por la posición del decoy. Por ello, es indispensable conocer las siguientes estrategias básicas:

R: definida como una opción que es ligeramente más débil que el target en su atributo más débil

F: se refiere a una opción que incrementa la frecuencia del atributo en el que el target es superior

RF: es una opción que combina $\mathbf{R}$ y $\mathbf{F}$ 
$\mathbf{R}^{*}$ : representa la versión extrema de $\mathbf{R}$

Tabla 1. Conjuntos de elección para diferentes estrategias decoy

\begin{tabular}{lcc}
\hline \hline & $\begin{array}{c}\text { Price/ } \\
\text { sixpack }\end{array}$ & $\begin{array}{c}\text { Quality } \\
\text { rating }\end{array}$ \\
\hline Range increasing (R) & & \\
$\quad$ Target & $\$ 1.80$ & 50 \\
Competitor & $\$ 2.60$ & 70 \\
$\quad$ Added decoy & $\$ 1.80$ & 40 \\
Extreme range increasing ( $\left.{ }^{*}\right)$ & & \\
$\quad$ Target & $\$ 1.80$ & 50 \\
$\quad$ Competitor & $\$ 2.60$ & 70 \\
$\quad$ Added decoy & $\$ 1.80$ & 30 \\
Frequency increasing (F) & & \\
$\quad$ Target & $\$ 1.80$ & 50 \\
$\quad$ Competitor & $\$ 2.60$ & 70 \\
$\quad$ Added decoy & $\$ 2.20$ & 50 \\
Range-frequency (RF) & & \\
$\quad$ Target & & \\
$\quad$ Competitor & $\$ 1.80$ & 50 \\
Added decoy & $\$ 2.60$ & 70 \\
\hline
\end{tabular}

Fuente: (Huber, Payne, \& Puto, 1982)

En la Tabla 1 se presentan ejemplos de cada estrategia, utilizando sixpacks de cerveza como opciones de elección.

\section{MATERIALES Y MÉTODOS}

La investigación se clasificó de tipo experimental cuyo objetivo principal fue explicar cómo las personas toman decisiones no solo en base a incentivos económicos, sino que también se ven influenciadas por otros aspectos, un señuelo o Decoy, por ejemplo.

\section{Grupos control y experimental}

Hay dos grupos en el experimento, los cuales son idénticos excepto porque uno incluye un señuelo y el otro no. El grupo que no incluye el señuelo se llama grupo experimental, mientras que el que lo incluye se denomina grupo control. El grupo control proporciona la base que nos permite ver si el experimento tiene algún efecto.

El factor que es diferente entre el grupo experimental y el control (en este caso, la presencia de la opción Decoy) se conoce como variable independiente. Esta variable es independiente 
porque no depende de lo que pase en el experimento. De hecho, es algo que los investigadores añadieron al experimento.

En contraste, la variable dependiente en este experimento es la respuesta que se mide para ver si el tratamiento tuvo algún efecto, que en este caso es la frecuencia obtenida por el target. Es decir, hay una opción "objetivo", la misma que debe ser elegida el mayor número de veces. La variable dependiente depende de la variable independiente (en este caso, el señuelo) y no al revés.

Debido al potencial de variación que pueden tener, los experimentos en economía necesitan un tamaño muy grande de muestra y, de manera ideal, repetirse varias veces. El tamaño de muestra se refiere al número de individuos puestos a prueba en el experimento, en este caso los 100 individuos por grupo. Una muestra más grande y varias repeticiones del experimento hacen que sea menos probable que lleguemos a una conclusión errónea debido a la variación aleatoria.

En algunos casos, no hay ninguna manera en que se pueda comprobar una hipótesis por medio de un experimento controlado (ya sea por razones prácticas o éticas). En ese caso, un científico puede poner a prueba la hipótesis haciendo predicciones sobre patrones que deberían verse en la naturaleza si la hipótesis es correcta. Entonces, puede recopilar datos para ver si el patrón realmente está allí. Con tal fin, se aplicó un experimento controlado sobre el funcionamiento

También se pretende demostrar que muy probablemente los experimentos en economía sean el método más idóneo para la enseñanza de esta ciencia social.

Como se mencionó anteriormente, la muestra estuvo conformada por 100 individuos elegidos aleatoriamente, con características muy similares: son mayores de edad y poseen conocimientos elementales de economía. Esto último, dado que para participar en el experimento resultaba imprescindible entender la relación entre riesgo y rentabilidad.

Para explicarlo, se organizaron dos escenarios donde primero se presentó un portafolio de inversión a los 100 individuos que, en este caso, son potenciales compradores. Luego, en un siguiente escenario, los mismos participantes recibieron otro portafolio con distintas alternativas. Así, por ejemplo: 
- Grupo Experimental (sin señuelo): aquí los individuos se enfrentan a solo dos alternativas de inversión, es decir, no hay un señuelo que desvíe su atención al momento de tomar una decisión.

- Grupo de Control (con señuelo): en este escenario los individuos se enfrentan a tres alternativas de inversión. A este respecto, el señuelo o Decoy, que es la opción de inversión añadida, supone un cambio determinante en el portafolio y la misma decisión de inversión.

\section{Antecedentes}

En el Curso de Nivelación de Carrera de la Universidad Técnica de Machala se imparten dos cátedras que son consideradas de tronco común. Hace algunas temporadas atrás, en una de ellas, denominada "Desarrollo de Habilidades del Pensamiento" (actualmente conocida como Universidad y Buen Vivir) se confirmó que la comparación es un proceso básico del pensamiento, por excelencia.

En su última temporada (Primer Semestre del 2019), como parte de sus actividades académicas, los investigadores habían contribuido en la realización de varios Proyectos Integradores de Saberes, los mismos que se habían convertido en máxima de todos los semestres. Ahora bien, dado que uno de los capítulos aborda la dimensión económica del proyecto, pudo detectarse que las ideas de negocio o emprendimientos desarrollados por los estudiantes, generalmente, enfrentaban problemas al momento de entender relaciones muy básicas como: costo-beneficio, oferta-demanda, entre los principales. Por tanto, varios proyectos quedaban archivados y no llegaban a ejecutarse. $O$, en su defecto, si se ejecutaban, terminaban fracasando.

Los docentes (investigadores) decidieron atender la problemática a través de un pequeño experimento fuera de clase:

Ciertamente, hay muchas personas interesadas en invertir pero que son aversas al riesgo, no solo en términos económicos, sino también en otros ámbitos. Más aún, cuando la información es escasa. Bajo estas condiciones, resulta naturalmente muy difícil hacer comprender que cierta alternativa de inversión es mejor o peor que otra. Pero eso está empezando a cambiar, aunque falte mucho por hacer.

En este sentido, la economía del comportamiento surge como el campo idóneo para experimentar e influir en la toma de decisiones.

ISSN: 1390-9320, Edición Especial, diciembre 2019 
Parafraseando a Peter Drucker: "Si no se mide lo que se hace, probablemente se pueda controlar, y si se puede controlar, muy probablemente se pueda mejorar". (Coffeen, 2009)

Entonces, ¿cómo introducir fundamentos sobre finanzas, marketing y economía simultáneamente?

\section{ANÁLISIS DE LOS RESULTADOS}

Lugar: "Campus Machala" de la Universidad Técnica de Machala

Objetivo: explicar cómo las personas raramente toman decisiones en términos absolutos, así como también obtener una demanda mayoritaria del stock de inversión A (target)

Tabla 2. Portafolio de inversión 1 - Alternativas

¿Cuál de las siguientes alternativas de inversión elegirías?

\begin{tabular}{|c|c|c|}
\hline & A & B \\
\hline $\begin{array}{c}\text { RIESGO } \\
\text { (pérdida esperada) }\end{array}$ & $2,66 \%$ & $2 \%$ \\
\hline $\begin{array}{c}\text { RENTABILIDAD } \\
\text { (ganancia esperada) }\end{array}$ & $0,33 \%$ & $0,17 \%$ \\
\hline
\end{tabular}

Fuente: Elaboración propia a partir de Google Forms 
Figura 5. Portafolio de inversión 1 - Decisiones

\section{¿Cuál de las siguientes alternativas de inversión elegirías?}

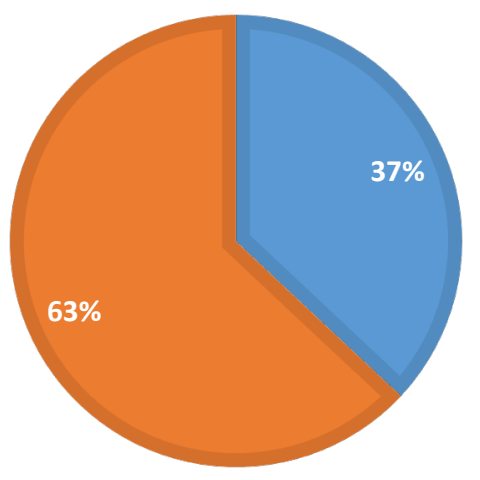

-Opción A

Fuente: Elaboración propia a partir de Google Forms/Encuesta estudiantes

Las preferencias para el Portafolio de inversión 1 nos muestran que solo hubo 37 participantes que eligieron la primera alternativa (opción A), mientras que 63 se decidieron por la segunda (opción B).

En promedio, podría afirmarse que los individuos participantes son aversos al riesgo, a pesar de tratarse de una simulación; dado que su demanda por la opción A es relativamente baja. De hecho, a pesar de no existir una gran diferencia entre el riesgo de ambas alternativas, apenas un $37 \%$ decidió optar por ese 0.33 de rentabilidad que ofrecía la opción A y que, por cierto, casi duplicaba los retornos de inversión de la opción B.

Tabla 3. Portafolio de inversión 2 - Alternativas

¿Cuál de las siguientes alternativas de inversión elegirías?

\begin{tabular}{|c|c|c|c|}
\hline & A & B & C \\
\hline $\begin{array}{c}\text { RIESGO } \\
\text { (pérdida esperada) }\end{array}$ & $2,66 \%$ & $2 \%$ & $2,67 \%$ \\
\hline RENTABILIDAD (ganancia esperada) & $0,33 \%$ & $0,17 \%$ & $0,25 \%$ \\
\hline
\end{tabular}

Fuente: Elaboración propia a partir de Google Forms 
Figura 6. Portafolio de inversión 2 - Decisiones

\section{¿Cuál de las siguientes alternativas de inversión elegirías?}

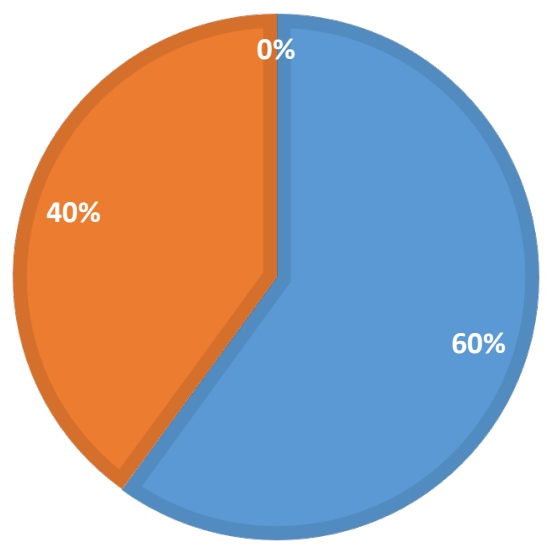

-Opción A

-Opción B

-Opción C

Fuente: Elaboración propia a partir de Google Forms/Encuesta estudiantes

En este nuevo escenario de inversión, se obtuvo algo muy parecido al gráfico anterior. La única diferencia es que las preferencias se invirtieron.

Así pues, se encontró que el 60\% prefería la primera alternativa (opción A), mientras que un $40 \%$ se decantó por la segunda (opción B). Es evidente que ese cambio se debió a la incorporación de una tercera alternativa (opción C), la cual era asimétricamente dominante.

Esta última (opción C) tenía un riesgo y rentabilidad superior que la "preferida" B, es decir, era inferior en algunos aspectos y superior en otros (peor riesgo-mejor rentabilidad); en comparación con la opción A, respecto de la cual era inferior en todos los aspectos (peor riesgo-peor rentabilidad). Por tanto, nadie eligió esa alternativa.

En otros términos, esta situación condujo a que unos cuantos se mantengan con la rentabilidad de 0.17 , muy probablemente porque les parecía una manera más segura de ganar dinero. Otros, a su vez, decidieron que una rentabilidad de 0.33 es más atractiva que las demás, independientemente del riesgo.

Lo cierto es que la incorporación de esta tercera opción produjo una variación que puede considerarse medianamente significativa $(\Delta>20 \%)$ en las preferencias de los inversionistas potenciales. Todo esto, a pesar de que la evidencia empírica ha confirmado que la mayoría de las personas se caracterizan por ser aversas al riesgo. 


\section{CONCLUSIONES Y RECOMENDACIONES}

Ha sido evidente que cuando alguien se enfrenta a una decisión entre tres alternativas, y dos de ellas son muy semejantes, tenderá a inclinarse por la mejor de aquellas dos. Con este mismo principio, si lo que pretendemos es posicionar un nuevo producto en el mercado y tenemos que competir con otros productos para los clientes, lo mejor es sacar una versión levemente desmejorada del producto que actuará como señuelo y dirigirá todas las miradas hacia el producto target (opción A). En definitiva, las personas no realizan elecciones en términos absolutos, sino que seleccionan una cosa en relación con las alternativas posibles. Esto es apenas una pequeña muestra de lo que podría ser una gran iniciativa pública y privada.

Así pues, ha sido notable que, en la mayor parte de los casos, los individuos se ven influenciados por incentivos al momento de tomar decisiones, independientemente si son estímulos económicos o sociales. Indudablemente, el experimento utilizado ha permitido reforzar conceptos clave tales como las estrategias de mercado, teoría financiera, así como la importancia del incentivo en la toma de decisiones. Por tanto, se concluye que para una mejor introducción de economía bien podría tomarse a la economía experimental como la base de la enseñanza, que, además, debe estar reforzada por los postulados de pensamiento económico, con el fin de dar diversos argumentos válidos a los estudiantes.

También es posible refutar el modelo del Homo economicus expuesto hace casi 150 años por la Escuela Neoclásica. Ciertamente, los seres humanos se caracterizan por su racionalidad, pero cabe destacar que también son previsiblemente irracionales, al menos en condiciones de mercado.

Por lo expuesto anteriormente, una recomendación fundamental sería que tanto gobiernos como instituciones de educación superior implementen "The Behavioral Insights Unit", esto es, la Unidad de Percepción del Comportamiento, como factor clave para mejorar la puesta en marcha de políticas públicas y la provisión de servicios.

\section{REFERENCIAS BIBLIOGRÁFICAS}

Chamberlin, E. H. (1948). An Experimental Imperfect Market Journal of Political Economy. 56(2), 95-108. 
Coffeen, L. (20 de Marzo de 2009). MarketCulture Blog. Recuperado de https://blog.marketculture.com/2009/03/20/if-you-cant-measure-it-you-cant-manageit-peter-drucker/

Encyclopedia Britannica, M. (1991). Knowledge in Depth. Chicago: The University of Chicago Press.

Fatás, E., \& Roig, J. (2004). Una introducción a la metodología experimental en economía. Cuadernos de Economía, 10-11.

García, L. A., \& Carreño, D. F. (2012). Economía experimental: un panorama general. Lebret, 280.

González-Prieto, D., \& Lordan, O. (2013). Influencia de efectos contextuales y rasgos psicológicos sobre. Intangible Capital, 9(1), 344.

Huber, J., Payne, J. W., \& Puto, C. (1982). Adding Asymmetrically Dominated Alternatives: Violations of Regularity and the Similarity Hypothesis. The Journal of Consumer Research, 9(1), 90-93.

Kahneman, D,. E Tversky A. (1979). Prospect Theory: An Analysis of Decisions Under Risk, Econometrica, 47, pp. 313-327.

Kahneman D. Frederick, S. (2002). Representativeness revisited: attribute subsitution on intutitive judgment, New York, Cambridge University Press

Kahneman, D (2002). Maps of Bounded Rationality: A perspective on intuitive judgment and choice. Prize Lecture. Nobel Foundation

Kaptein, M. C., Emden, R. V., \& Lanuzzi, D. (2016). Tracking the decoy: maximizing the decoy effect through sequential experimentation. Palgrave Communications, 3.

Pascale, R., \& Pascale, G. (2007). Ciencias Psicológicas, 1(2), 166.

Pascale, R., \& Pascale, G. (2007). TOMA DE DECISIONES ECONÓMICAS: EL APORTE COGNITIVO EN LA RUTA DE SIMON, ALLAIS Y TVERSKY Y KAHNEMAN. Ciencias Psicológicas, 1(2), 161-162.

Plott, C. R., \& Smith, V. L. (2008). The Handbook of Experimental Economics Results (Vol. 1). (N. Holland, Ed.) New York.

Samson, A. (2014). The Behavioral Economics Guide 2014. London. Obtenido de https://www.behavioraleconomics.com/BEGuide2014.pdf

ISSN: 1390-9320, Edición Especial, diciembre 2019 
Samuelson, P. A., \& Nordhaus, W. D. (1985). Economics. New York: McGraw-Hill.

Smith, V. (1962). An Experimental Study of Competitive Market. The Journal of Political Economy, 111-137.

Smith, V. (2005). ¿Qué es la Economía Experimental? Apuntes del CENES, 12.

Stiglitz, J. (1993). Economía. Barcelona: Ariel Economía.

Sugden, R. (2009). On Nudging: A Review of Nudge: Improving Decisions About Health, Wealth and Happiness by Richard H. Thaler and Cass R. Sunstein. International Journal of the Economics of Business, 16(3), 366.

Tlapanco, H. D. (2016). Experimentos en una ciencia no experimental. Investigación Económica, 75(295), 59. 